\title{
In-line-Monitoring der Elektrolyt- und Harnstoffkonzentration in extrakorporalen Kreisläufen zur Individualisierung der Nierenersatztherapie
}

\author{
Marc Berger, Stefan Zimmermann \\ Leibniz Universität Hannover, Institut für Grundlagen der Elektrotechnik und Messtechnik, \\ Fachgebiet Sensorik und Messtechnik, Appelstr. 9A, 30167 Hannover, Deutschland \\ berger@geml.uni-hannover.de
}

\begin{abstract}
Zusammenfassung
Bei akuten nephrologischen Notfällen ist es notwendig, dass die Funktion der Niere durch eine Nierenersatztherapie bzw. Akutdialyse übernommen wird. Bei dieser werden Giftstoffe über eine semipermeable Membran, mit Blut auf der einen Seite der Membran und einem sogenannten Dialysat auf der anderen Seite, entfernt und der Elektrolythaushalt reguliert. Da der Transport der Giftstoffe und der Elektrolyte über die semipermeable Membran per Diffusion erfolgt, ist der Konzentrationsgradient zwischen Blut und Dialysat maßgebend für die Stoffaustauschgeschwindigkeit. Ein zu schnelles oder überschüssiges Absenken der Elektrolyte kann dabei für den Patienten sehr belastend sein und mitunter lebensbedrohliche Zustände hervorrufen. Daher besteht der Bedarf, die Nierenersatztherapie bei der Akutdialyse auf die individuellen Bedürfnisse des Patienten einzustellen. Beispielsweise kann durch gezieltes Verändern der Dialysatkonzentration der Konzentrationsgradient über der Membran und so die Stoffaustauschgeschwindigkeit gesteuert werden. Hierzu ist es jedoch notwendig, die Elektrolytkonzentration im Blut kontinuierlich zu überwachen. Darüber hinaus soll eine OnlineÜberwachung der Harnstoffkonzentration im Blut erfolgen, um die Effektivität der Dialyse zu beurteilen. In dieser Arbeit wird daher ein neuartiger Ansatz für ein kontinuierliches In-line-Monitoring der Elektrolyt- und Harnstoffkonzentration in extrakorporalen Kreisläufen zur Individualisierung der Nierenersatztherapie vorgestellt. Mit einem ersten Demonstrator wurde das Konzept experimentell hinsichtlich Einschwingverhalten und Messabweichung untersucht. Die Messabweichung, bei einer für die Akutdialyse typischen Änderungsgeschwindigkeit der Konzentration von $10 \%$ pro Stunde, beträgt nur 2,2 \%. Ferner wurde in computergestützten Simulationen der Haupteinflussfaktor identifiziert, um das dynamische Verhalten weiter zu verbessern.
\end{abstract}

Keywords: In-line-Monitoring, Akutdialyse, elektrochemische Sensoren, ionenselektive Elektroden, Individualisierung der Dialyse, Blutparameterbestimmung

\section{Einleitung}

Bei der Dialyse handelt es sich um ein Blutreinigungsverfahren, welches zum Einsatz kommt, wenn die Nierenfunktion teilweise oder vollständig ausfällt. Im Falle eines akuten Nierenversagens, welches in der Regel durch eine schwerwiegende Grunderkrankung (wie z. B. Sepsis, Multiorganversagen oder Kreislaufinsuffizienz) hervorgerufen wird, ist eine Akutdialyse erforderlich. Die Behandlungsdauer beträgt in der Regel etwa 72 Stunden. Hierdurch wird das kurz- bis mittelfristige Überleben des betroffenen Patienten sichergestellt [1, 2]. Dabei werden aufgenommene oder vom Körper gebildete Stoffe, welche sonst überwiegend oder ausschließlich über die Niere ausge- schieden werden, aus dem Blut per Diffusion über eine semipermeable Membran entfernt [3]. Somit hängt die Geschwindigkeit des Stoffaustausches, zwischen Blut auf der einen und Dialysat auf der anderen Seite der Membran, maßgeblich vom Konzentrationsgradienten eines Stoffes ab. Wird die Serumkonzentration eines Stoffes, im Speziellen der Elektrolyte, während der Dialyse zu schnell oder überschüssig gesenkt, kann dies gesundheitsschädliche Folgen für den Patienten haben $[1,4]$. Insbesondere durch die ohnehin schon vorhandene Grunderkrankung ist jegliche zusätzliche Belastung des Patienten zu vermeiden. Die Dosierung der Nierenersatztherapie erfolgt derzeit anhand der klinischen Einschätzung des behandelnden Arztes und von 
Laborparametern, welche typischerweise täglich oder zumindest vor Dialysebeginn erhoben werden $[1,2]$. Diese liegen durch die zeitaufwendige Laboranalyse jedoch verspätet und nur zu diskreten Zeitpunkten vor. Hierdurch ist es nicht möglich, die Elektrolytkonzentration des Dialysats auf die individuellen Bedürfnisse des Patienten anzupassen, um einen optimalen Konzentrationsgradienten zwischen Blut und Dialysat einzustellen und eine möglichst schonende Behandlung zu erhalten. Darüber hinaus ist die Reduktion der Harnstoffkonzentration ein wichtiger Marker, um die Effektivität der Dialyse zu beurteilen. Daher wird im Rahmen dieser Arbeit ein neuer und instrumentell einfacher Ansatz zur In-line-Überwachung der Elektrolyt- und Harnstoffkonzentration in extrakorporalen Kreisläufen zur Individualisierung der Nierenersatztherapie vorgestellt.

\section{Konzept}

Ein gängiges Verfahren zur Bestimmung der Elektrolytkonzentrationen in flüssiger Phase ist die potentiometrische Messung mithilfe von ionenselektiven Elektroden (ISE). Hierbei wird eine konzentrationsabhängige Spannung zwischen der ISE und einer Referenzelektrode gemessen. Die Harnstoffkonzentration kann mithilfe eines enzymatischen Biosensors bestimmt werden. Hierzu wird z. B. das Enzym Urease auf einer ammonium- oder ammoniakselektiven Elektrode immobilisiert. Der Harnstoff wird in wässrigen Lösungen unter katalytischer Wirkung des Enzyms Urease in Ammoniumionen respektive Ammoniak gespaltet [5-7]. Wird die Urease dicht vor dem sensitiven Bereich der entsprechenden Elektrode immobilisiert, stellt sich eine harnstoffkonzentrationsabhängige Spannung zwischen ISE und Referenzelektrode ein.

Das direkte In-line-Messen mit ISE im Blut ist aus Gründen der Hämokompatibilität jedoch nicht möglich. Durch das Ablagern von z. B. Proteinen auf der ionenselektiven Membran der ISE kommt es zum sogenannten Membranfouling [8]. Dieses führt zu einer zeitlichen Drift der Messspannung zwischen ISE und Referenzelektrode und im schlimmsten Fall zur Initiierung der Blutgerinnung. Aus diesem Grund wird hier der Ansatz verfolgt, die ISE in einem mit physiologischer Elektrolytlösung gefülltem Kompartiment zu platzieren, welches durch eine Dialysemembran vom Blutfluss im extrakorporalen Kreislauf getrennt ist (siehe Abb. 1).

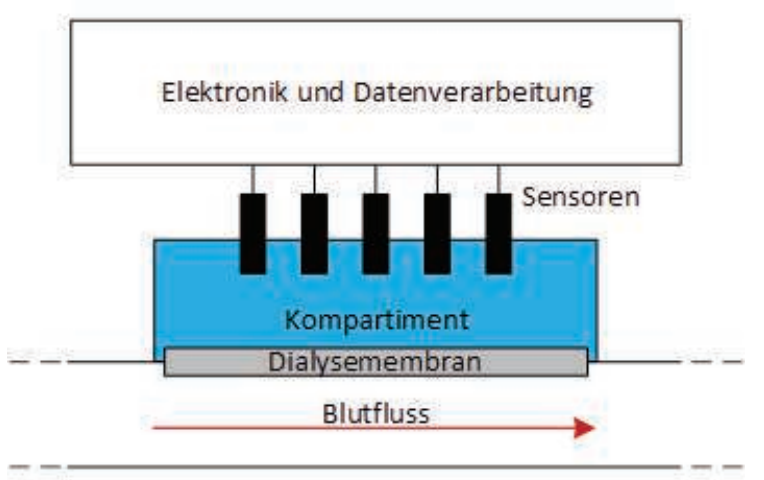

Abb. 1: Schematische Darstellung des Inline-Konzeptes zur Bestimmung von Blutparametern.

Durch die Trennung von Blut und flüssigkeitsgefülltem Sensorkompartiment stehen die verwendeten Sensoren nicht mehr im direkten Kontakt mit dem Blut. Sowohl die Elektrolyte als auch Harnstoff können durch die Dialysemembran diffundieren und nach kurzer Zeit stellt sich ein Konzentrationsgleichgewicht zwischen Blut und der Flüssigkeit im Kompartiment ein. Stoffe, welche größer als die Trenngrenze der Membran sind, werden von dieser zurückgehalten und kommen nicht in Kontakt mit den Sensoren. Hierunter fallen z. B. Eiweiße und Zellen. Dieser Ansatz ist hämokompatibel und verhindert die Ablagerung von z. B. Proteinen auf der der ISE.

\section{Simulationen}

Um das zeitliche Verhalten dieses In-lineMesskonzeptes bewerten zu können, wurde ein zweidimensionales Simulationsmodell mithilfe der Software Comsol Multiphysics 5.1 erstellt. Abb. 2 zeigt den schematischen Aufbau des Simulationsmodells. Im unteren Bereich fließt das Blut des extrakorporalen Kreislaufs tangential an der Dialysemembran entlang. Aus Gründen der Hämokompatibilität ist eine möglichst laminare Blutströmung zwingend erforderlich und die Strömungsgeometrie des Sensorsystems ist später entsprechend zu designen. Für die Simulation wird daher ein parabolisches Strömungsprofil angenommen. Die Dialysemembran besitzt eine Stärke von $28 \mu \mathrm{m}$. Über der Membran befindet sich das flüssigkeitsgefüllte Kompartiment mit einem Durchmesser $d_{k}$ von 12,1 mm und einer Höhe $h_{k}$ von $5 \mathrm{~mm}$. Jede ISE besitzt einen Durchmesser $d_{s}$ von $12 \mathrm{~mm}$ und wird von oben in das jeweilige Kompartiment eingeführt. Zwischen der ISE und der Dialysemembran stellt sich der Abstand $h_{s}$ ein. 


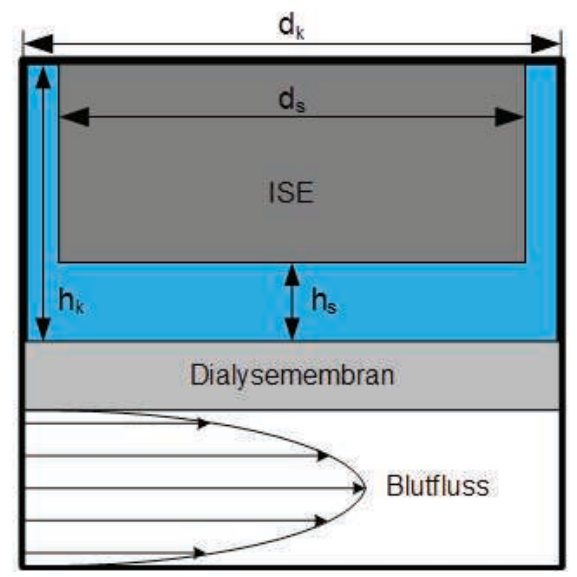

Abb. 2: Schematische Darstellung des Simulationsmodells.

Zu Beginn der Simulation wird die Konzentration im extrakorporalen Kreislauf sprunghaft von null auf einen Wert $\mathrm{C}_{0}$ angehoben. In der Realität treten i.d.R. keine sprunghaften Konzentrationsänderungen auf. Die Anfangselektrolytkonzentration im Kompartiment wird in der Simulation zudem auf null gesetzt, was einer Anfangsfüllung des Kompartiments mit reinem Wasser entspricht und zu deutlich größeren absoluten Messabweichungen zu Messbeginn führt als eine Füllung mit physiologischer Elektrolytlösung. Das Blut strömt aufgrund des konvektiven Transports tangential an der Dialysemembran entlang. Abb. 3 zeigt einen Ausschnitt des Simulationsmodells zu Beginn der Simulation. Der Farbverlauf stellt die Höhe der Konzentration eines Stoffes dar. Rot steht hierbei für eine hohe Konzentration und blau für eine geringe. Innerhalb des extrakorporalen Kreislaufs dominiert der Stofftransport aufgrund von Konvektion. Durch die Dialysemembran und innerhalb des Kompartiments findet dieser jedoch ausschließlich aufgrund von Diffusion statt. Für den Diffusionskoeffizient $\mathrm{D}$ in der Membran und im Kompartiment wurde in der Simulation $D=2,5 \cdot 10^{-9} \mathrm{~m}^{2} / \mathrm{s}$ gewählt [9].

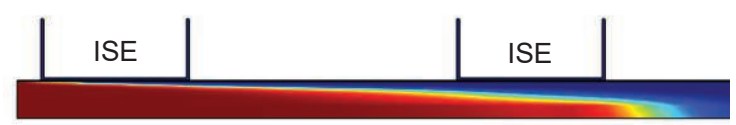

Abb. 3: Ausschnitt aus dem Simulationsmodell zu Beginn der Simulation. Der Farbverlauf stellt die Höhe der Konzentration eines Stoffes dar. Rot steht für eine hohe Stoffkonzentration und blau für eine geringe.

Aufgrund des diffusiven Stofftransportes kommt es zu einem Konzentrationsausgleich zwischen Blut und der Flüssigkeit im Kompartiment, wobei lediglich Stoffe ausgetauscht werden, die kleiner als die Trenngrenze der
Membran sind. Abb. 4 zeigt einen Ausschnitt des Simulationsmodells nach $450 \mathrm{~s}$ bei zwei verschiedenen Abständen $\mathrm{h}_{\mathrm{s}}$ zwischen der ISE und der Dialysemembran. Links beträgt $h_{s}=3,5 \mathrm{~mm}$ und rechts ist $h_{s}=1,7 \mathrm{~mm}$. Wie anhand von Abb. 4 zu erkennen, ist die Konzentration in beiden Kompartimenten nach $450 \mathrm{~s}$ bereits gestiegen. Durch das geringere Kompartimentvolumen bei kleineren Werten von $h_{s}$ ist der Konzentrationsausgleich rechts jedoch deutlich weiter fortgeschritten.
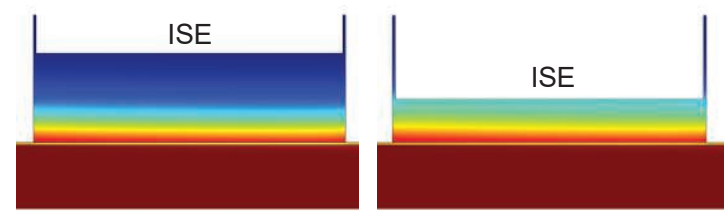

Abb. 4: Vergleich der Kompartimentkonzentrationsverteilung bei einem Abstand $h_{s}=3,5 \mathrm{~mm}$ (links) und $h_{s}=1,7 \mathrm{~mm}$ (rechts) nach $450 \mathrm{~s}$.

Der zeitliche Verlauf der Konzentration innerhalb des Kompartiments, in unmittelbarer Nähe der ISE, für die Abstände $h_{s}=3,5 \mathrm{~mm}$ (rot) und $\mathrm{h}_{\mathrm{s}}=1,7 \mathrm{~mm}$ (blau), bei sprunghafter Änderung der Stoffkonzentration im extrakorporalen Kreislauf auf den Wert $\mathrm{C}_{0}$, ist in Abb. 5 dargestellt. Der Konzentrationsverlauf wurde dabei auf $\mathrm{C}_{0}$ normiert.

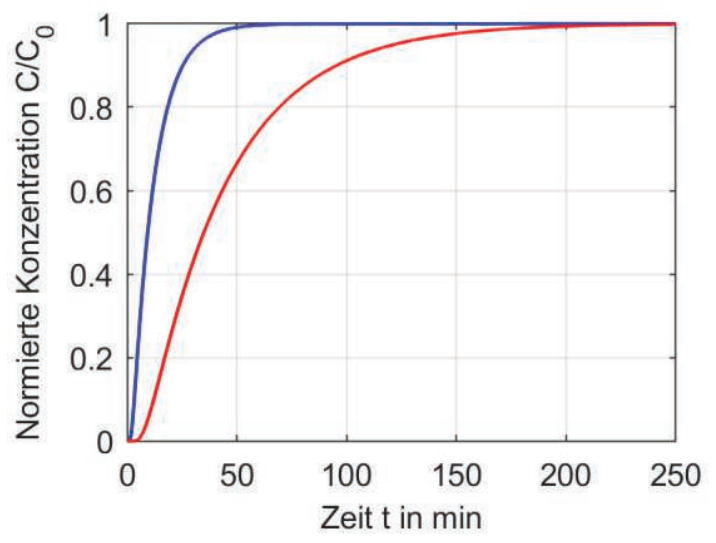

Abb. 5: Vergleich des zeitlichen Konzentrationsverlaufs bei $h_{s}=3,5 \mathrm{~mm}$ (rot) und $h_{s}=1,7 \mathrm{~mm}$ (blau) normiert auf die Blutkonzentration $\mathrm{C}_{0}$.

Anhand von Abb. 5 ist zu erkennen, dass der Abstand $h_{s}$ einen erheblichen Einfluss auf die Geschwindigkeit des Konzentrationsausgleichs hat. Das Messsystem ist demnach bezüglich kleiner $h_{s} z u$ optimieren. So wird bei einem Abstand von $h_{s}=3,5 \mathrm{~mm}$ für einen Konzentrationsausgleich von $90 \%$ zwischen Blut und Kompartiment eine lange Zeit von etwa 96 min benötigt. Bei $h_{s}=1,7 \mathrm{~mm}$ sind es dagegen nur noch etwa 25,5 min. Es sei hier noch einmal darauf hingewiesen, dass hierdurch eine an- 
genommene sprunghafte Konzentrationsänderung im Blut von null auf $\mathrm{C}_{0}$ und eine Anfangskonzentration im Kompartiment von null die systemtechnisch interessante Sprungantwort ermittelt wird. Während des Therapieverlaufs kommen i.d.R. keine sprunghaften Konzentrationsänderungen vor. Außerdem ist das Kompartiment später mit physiologischer Elektrolytlösung gefüllt, was zu deutlich kleineren absoluten Messabweichungen führt. Der Einfluss von $\mathrm{h}_{\mathrm{s}}$ ist ebenfalls in Abb. 6 zu erkennen. Hier wurde die benötigte Zeit t9o für einen Konzentrationsausgleich von $90 \%$ zwischen Blut und Kompartiment über dem Abstand $h_{s}$ aufgetragen.

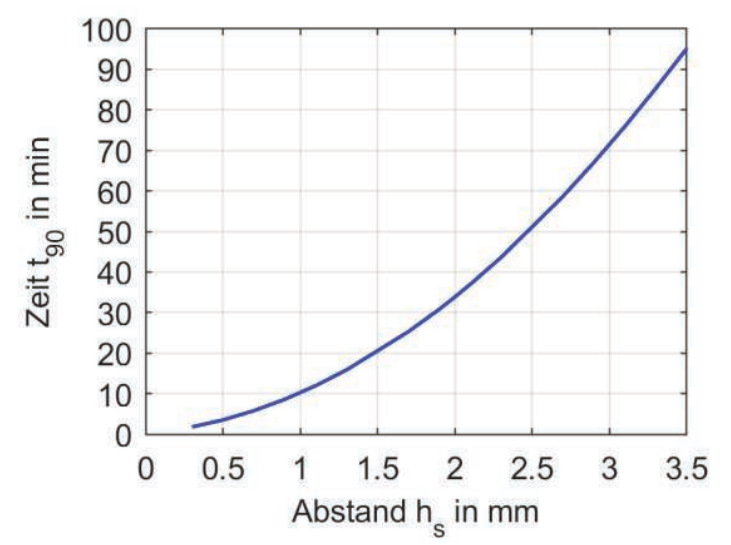

Abb. 6: Benötigte Zeit to für einen Konzentrationsausgleich von $90 \%$ zwischen Blut und Kompartiment als Funktion des Abstandes $h_{s}$ zwischen ISE und Dialysemembran.

Abb. 6 verdeutlicht, dass sich die Zeitdauer t90 durch Verringerung des Abstandes $h_{s}$ erheblich reduzieren lässt. Um jedoch einen direkten Kontakt des sensitiven Bereiches der ISE mit der Dialysemembran zu vermeiden, lässt sich $\mathrm{h}_{\mathrm{s}}$ in der Praxis nicht beliebig reduzieren. In einem ersten, hier vorgestellten Demonstrator beträgt der Abstand $h_{s}=1,7 \mathrm{~mm}$. Daher werden zunächst die Simulationsergebnisse für diesen Abstand weiter betrachtet.

Da bei der Akutdialyse beispielsweise die Natriumionenkonzentration $\mathrm{Na}^{+}$von einem bestimmten Anfangswert $\mathrm{C}_{0}$ typischerweise exponentiell mit etwa $10 \%$ pro Stunde abnimmt, nimmt die Eingangskonzentration im folgenden Simulationsmodell gleichermaßen ab und wird auf die Anfangskonzentration $\mathrm{C}_{0}$ normiert (siehe Abb. 7, schwarz gestrichelte Linie). Die Konzentration im Kompartiment folgt der Blutkonzentration nach einer Einschwingzeit von etwa $40 \mathrm{~min}$. Die anfängliche große Messabweichung ist die Folge des großen Konzentrationssprunges $\mathrm{C}_{0} \mathrm{zu}$ Beginn der Simulation, was nicht den realen Bedingungen entspricht (siehe Abb. 7, blaue Line).

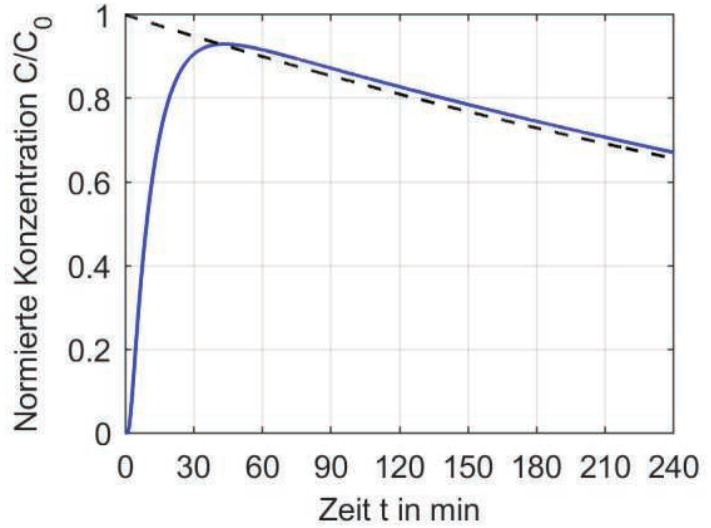

Abb. 7: Zeitlicher Verlauf der Kompartimentkonzentration bei einer exponentiell abnehmenden Blutkonzentration von $10 \%$ pro Stunde und einem Abstand $h_{s} 1,7 \mathrm{~mm}$.

Der sich ergebende relative Fehler $f$ zwischen Blut- und Kompartimentkonzentration bei einer Änderungsgeschwindigkeit von $10 \%$ pro Stunde beträgt nach der anfänglichen Einschwingzeit nur 2,2\% und ist in Abb. 8 dargestellt.

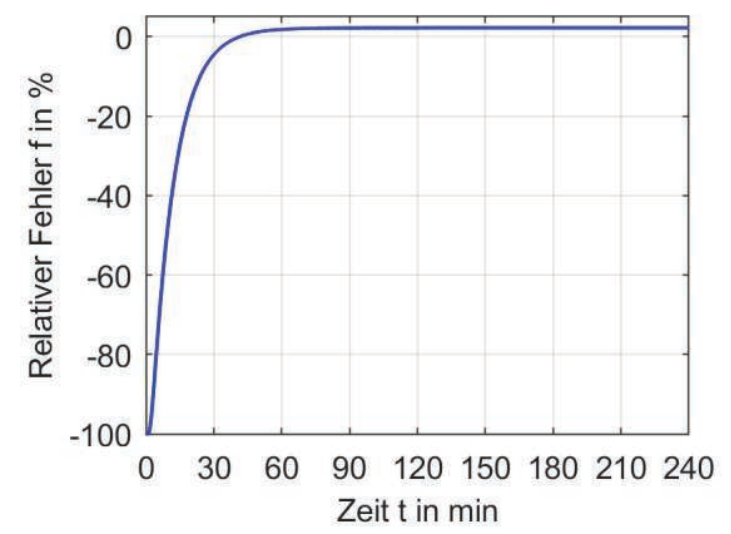

Abb. 8: Zeitlicher Verlauf des relativen Fehlers $f$ in \% zwischen Blut- und Kompartimentkonzentration bei einem Abstand $h_{s}=1,7 \mathrm{~mm}$.

Wie bereits in Abb. 6 verdeutlicht, werden die dynamischen Eigenschaften durch Verringerung des Abstandes $h_{s}$ verbessert. In Abb. 9 ist der Verlauf der Kompartimentkonzentration bei einem Abstand von $h_{s}=0,5 \mathrm{~mm}$ dargestellt (blaue Linie). Die Eingangskonzentration nimmt hier ebenfalls exponentiell mit $10 \%$ pro Stunde ab (schwarz gestrichelte Linie). Die Einschwingzeit beträgt durch die Reduktion des Abstandes nur noch 8,5 min. 


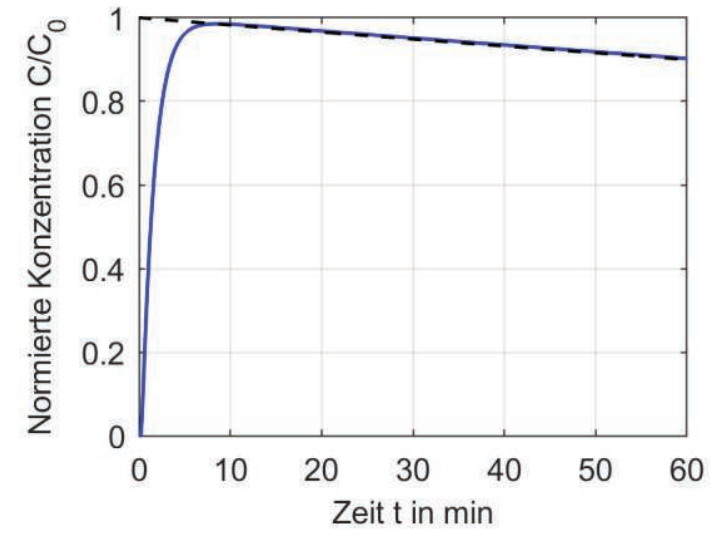

Abb. 9: Zeitlicher Verlauf der Kompartimentkonzentration bei einer exponentiell abnehmenden Blutkonzentration von $10 \%$ pro Sunde und einem Abstand $h_{s}=0,5 \mathrm{~mm}$.

Der relative Fehler reduziert sich hierdurch ebenfalls und ist in Abb. 10 dargestellt. Nach der Einschwingzeit stellt sich ein konstanter relativer Fehler von nur 0,3\% ein.

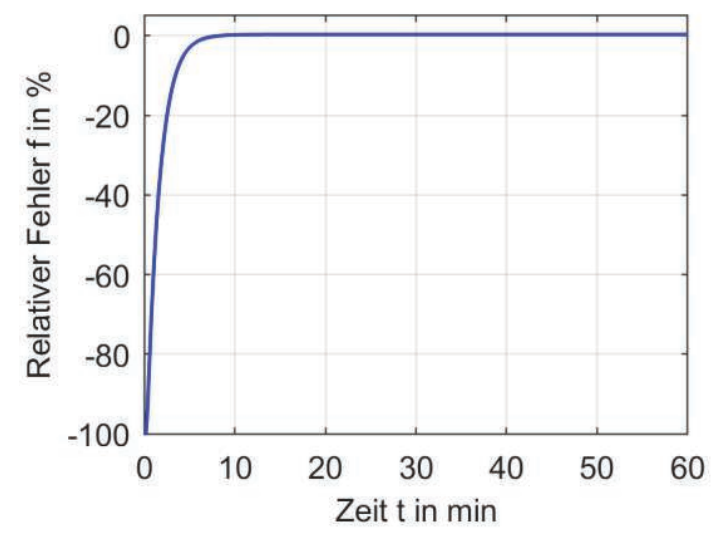

Abb. 10: Zeitlicher Verlauf des relativen Fehlers $f$ in \% zwischen Blut- und Kompartimentkonzentration bei einem Abstand $h_{s}=0,5 \mathrm{~mm}$.

Durch den geringen Anteil am Gesamtvolumen hat sich in weiteren Simulationen gezeigt, dass der Einfluss der Kompartimenthöhe $h_{k}$ einen vernachlässigbaren Einfluss auf das dynamische Verhalten und damit auf den resultierenden relativen Fehler hat.

\section{Experimenteller Aufbau und Messungen}

Zur Validierung der Simulationsergebnisse dient im Folgenden eine experimentelle Charakterisierung eines ersten Demonstrators. Dieser wurde aus dem thermoplastischen Kunststoff PEEK gefertigt. Der Demonstrator besteht aus zwei Teilen. Am unteren Teil befinden sich links und rechts zwei Anschlüsse für die Schläuche des extrakorporalen Kreis- laufsystems. Zwischen dem oberen und unteren Teil der Messkammer wird eine Dialysemembran aus regenerierter Cellulose, mit einer Stärke von $28 \mu \mathrm{m}$, eingespannt (Typ: RCTNatureFlex-NP, Reichelt Chemietechnik). Diese Membran besitzt eine Trenngrenze von etwa $10 \mathrm{kD}$ bis $20 \mathrm{kD}$. Im oberen Teil der Messkammer befinden sich die Plätze für die ISE und die Referenzelektrode. Des Weiteren befindet sich hier eine Nut, über welche das Kompartiment mit einer physiologischen Elektrolytlösung vor Messbeginn gefüllt werden kann.

Die erste Charakterisierung erfolgte mithilfe einer Natriumchloridlösung ( $\mathrm{NaCl})$. Hierzu wurden das Kreislaufsystem und das Kompartiment mit einer $\mathrm{NaCl}$-Lösung gefüllt und die Konzentration im Kreislaufsystem sprunghaft auf den Wert $\mathrm{C}_{0}$ angehoben. Die Bestimmung der Sprungantwort der Konzentration innerhalb des Kompartiments erfolgte mithilfe einer natriumselektiven Elektrode (Polymermembranelektrode $\mathrm{Na}$, Metrohm) gegen eine $\mathrm{Ag} / \mathrm{AgCl}$ Referenzelektrode (373/SSG/12, AMEL). Die konzentrationsabhängige Spannung zwischen den Elektroden wurde mithilfe des Potentiostaten EmStat3 4WE der Firma PalmSens aufgezeichnet. Der Abstand $h_{s}$ zwischen den Elektroden und der Dialysemembran beträgt bei dem ersten Demonstrator $1,7 \mathrm{~mm}$.

Der rote Verlauf in Abb. 12 zeigt die gemessene und per Savitzky-Golay-Filter geglättete Sprungantwort. Die Konzentration wurde auf $\mathrm{C}_{0}$ normiert. Es zeigt sich, dass Konzentrationsausgleich von $90 \%$ nach etwa 25,7 Minuten erreicht wird. Zum Vergleich ist das Simulationsergebnis der Sprungantwort bei gleicher Geometrie dargestellt (Abb. 12, blau gepunkteter Verlauf).

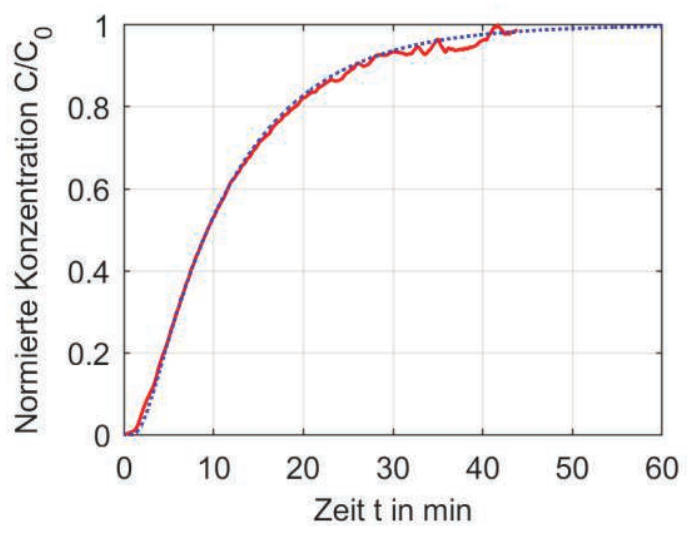

Abb. 12: Vergleich zwischen gemessener Sprungantwort der $\mathrm{Na}^{+}-$Konzentration im Kompartiment (rot) und der simulierten Sprungantwort (blau gepunktet) bei einem Abstand $h_{s}=1,7 \mathrm{~mm}$ normiert auf die $\mathrm{An}$ fangskonzentration $C_{0}$. 
Anhand von Abb. 12 ist zu erkennen, dass die gemessene Sprungantwort hervorragend mit der simulierten übereinstimmt. Somit können die Ergebnisse aus der Simulation für exponentiell abnehmender Konzentration von $10 \%$ pro Stunde im extrakorporalen Kreislauf übernommen werden. Demnach kann mit dem derzeitigen Demonstrator die Konzentration im Kreislaufsystem mit einem relativen Fehler von nur 2,2 \% bestimmt werden.

\section{Zusammenfassung}

In dieser Arbeit wird ein Konzept zur In-lineMessung der Elektrolyt- und Harnstoffkonzentration in extrakorporalen Kreisläufen zur Individualisierung der Nierenersatztherapie vorgestellt. Mit einem ersten Demonstrator ist es bereits möglich, bei einer exponentiell verlaufenden Konzentration in einer Testlösung im extrakorporalen Kreislauf mit einer Änderungsgeschwindigkeit von $10 \%$ pro Stunde, wie sie bei der Akutdialyse typischerweise vorkommt, mit einem relativen Fehler von nur 2,2 \% zu bestimmen. Ferner wurde ein Simulationsmodell experimentell validiert. Hierbei wurde festgestellt, dass das Simulationsmodell sehr gute Vorhersagen zum dynamischen Verhalten des Messsystems liefert. Eine Optimierung des dynamischen Verhaltens lässt sich insbesondere durch Minimierung des Abstandes $h_{s}$ zwischen ISE und Dialysemembran erreichen. Wird dieser Abstand beispielsweise von $h_{s}=1,7 \mathrm{~mm}$ auf $h_{s}=0,5 \mathrm{~mm}$ reduziert, verringert sich die Einschwingzeit etwa um einen Faktor 5 und der relative Fehler zwischen Blut und Kompartimentkonzentration sinkt von $2,2 \%$ auf $0,3 \%$. Aufgrund der längeren Behandlungsdauer bei der Akutdialyse, welche in der Regel etwa 72 Stunden beträgt, ist eine anfängliche Einschwingzeit im Minutenbereich vernachlässigbar.

\section{Danksagungen}

Gefördert durch das Bundesministerium für Bildung und Forschung (BMBF) unter dem Förderkennzeichen: 13GW0085B

\section{Literaturnachweis}

[1] P. J. Heering and M. Schmitz, "Akutes Nierenversagen auf der Intensivstation," Intensivmed, vol. 47, no. 6, pp. 429-433, 2010.

[2] R. Nowack, R. Birck, and T. Weinreich, Eds, Dialyse und Nephrologie für Fachpersonal, 3rd ed. Heidelberg: Springer, 2009.

[3] M. Sivalingam and K. Farrington, "Haemodialysis," Medicine, vol. 35, no. 8, pp. 461-465, 2007.
[4] F. Locatelli, V. La Milia, L. Violo, L. Del Vecchio, and S. Di Filippo, "Optimizing haemodialysate composition," (eng), Clinical kidney journal, vol. 8, no. 5, pp. 580-589, 2015.

[5] R. Koncki, "Recent developments in potentiometric biosensors for biomedical analysis," (eng), Analytica Chimica Acta, vol. 599, no. 1, pp. 7-15, 2007.

[6] G. Dhawan, G. Sumana, and B. D. Malhotra, "Recent developments in urea biosensors," Biochemical Engineering Journal, vol. 44, no. 1, pp. 42-52, 2009.

[7] M. SINGH, N. VERMA, A. GARG, and N. REDHU, "Urea biosensors," Sensors and Actuators B: Chemical, vol. 134, no. 1, pp. 345-351, 2008.

[8] M. K. Sharma, F. P. Wieringa, A. J. H. Frijns, and J. P. Kooman, "On-line monitoring of electrolytes in hemodialysis: on the road towards individualizing treatment," (eng), Expert review of medical devices, vol. 13, no. 10, pp. 933-943, 2016.

[9] P. W. Atkins and J. de Paula, Physikalische Chemie. Place of publication not identified: Wiley-VCH, 2014. 